\title{
Rank histograms and MVL diagrams for weather forecasting The use of Rank Histograms and MVL Diagrams to characterize ensemble evolution in weather forecasting
}

\author{
Jorge A. Revelli, Miguel A. Rodríguez* and Horacio S. Wio \\ Instituto de Física de Cantabria (UC and CSIC), \\ Avda. de los Castros, s/n, E-39005 Santander, Spain
}

(Dated: June 13, 2010)

\begin{abstract}
Rank Histograms are suitable tools to assess the quality of ensembles within an ensemble prediction system or framework. By counting the rank of a given variable in the ensemble, we are basically making a sample analysis, which does not allow us to distinguish if the origin of its variability is external noise or comes from chaotic sources. The recently introduced Mean to Variance Logarithmic (MVL) Diagram accounts for the spatial variability, being very sensitive to the spatial localization produced by infinitesimal perturbations of spatiotemporal chaotic systems. By using as a benchmark a simple model subject to noise, we show the distinct information given by Rank Histograms and MVL Diagrams. Hence, the main effects of the external noise can be visualized in a graphic. From the MVL diagram we clearly observe a reduction of the amplitude growth rate and of the spatial localization (chaos suppression), while from the Rank Histogram we observe changes in the reliability of the ensemble. We conclude that in a complex framework including spatiotemporal chaos and noise, both provide a more complete forecasting picture.
\end{abstract}

KEYWORDS: Rank histogram, $M V L$ diagram, ensemble evolution, noise, space-time chaos, forecasting.

\footnotetext{
* Corresponding Author: rodrigma@ifca.unican.es
} 


\section{Introduction}

It is well known that noise and chaos represent, respectively, two kinds of essentially different phenomena that, are present in many physical systems. Noise is induced by genuine stochastic sources, while chaos only shows pseudo-randomness that is deterministic in its origin (as an example of the pseudo-random behavior in chaotic systems see (Caroll and Pecora, 1993a, 1993b; Anishchenko et al., 1993)). Spatiotemporal chaos is intrinsically irregular in both space and time and represents a prototype of deterministic pseudo-randomness. In order to deepen our understanding of chaotic systems, it is interesting to see what would happen as a result of the interaction between these two sources of irregularities. Even more important is the effect of both phenomena in the predictability of general physical systems and in particular those involved in weather forecasting.

In the last decade weather forecasting has been the main driving force behind most of the studies performed on predictability, and the field in which the majority of techniques of practical use have been created. Such approach, the Ensemble Prediction System has been established as the most appropriate methodology to deal with the uncertainties of the atmospheric models used in weather forecasting (Gneiting and Raftery, 2005). An ensemble provides a probabilistic forecast which comprises multiple runs of one or several numerical weather models. Uncertainties due to models can be classified as external noise, whereas uncertainties due to initial conditions come from the chaotic effect of amplification of small perturbations. In a realistic representation of the atmosphere both aspects must be taken into account and it is important to quantify the contribution of each, in order to optimize the models, the ensemble structure, and the choice of the initial conditions. In this paper we show how the recently introduced Mean to Variance Logarithmic Diagram (MVL) (Gutiérrez et al., 2008) allows us to distinguish between these kinds of variability and is thus a useful tool for weather prediction.

In standard methods of ensemble forecasting the evolution of initial errors is usually assessed by root-mean-square (RMS) errors or other methods based on sample statistics to measure the spread of ensemble members. These methods are convenient to quantify uncertainties in general, but they are not sensitive to the origin of the noise (Kalnay, 2002). Recently, some studies have focused on the spatiotemporal analysis of perturbations to provide us the possibility of using statistical indicators sensitive to the chaotic origin of determin- 
istic noise (López et al.,2004). It is known that spatial chaos in its infinitesimal evolution leads to localized perturbations due to the relaxation towards the attractor (Pikovsky and Politi, 1998), but the way in which the noise affects this process is not obvious (Gottwald and Melbourne, 2005). Hence, to study complex systems with chaotic behavior and noise effects, both points of view are necessary.

Here we exemplify this assertion by comparing the information obtained from standard Rank Histograms (RH) and MVL diagrams in a simple model of spatiotemporal chaos subject to noise. We use the Lorenz'96 model (Lorenz, 1996; Lorenz and Emanuel, 1998), with an external noisy forcing, i.e. the model takes into account two kinds of perturbations, a deterministic perturbation given by the chaotic behavior of the model and a stochastic one which we have assumed as an effective way of including other "uncontrolled" contributions for a more realistic evolution. The relevance of this model rests on the fact that it represents a simple but still realistic description of some physical properties of global atmospheric models.

This work is organized as follows: in section 2 we describe the Lorenz'96 model and we introduce the ensemble prediction system. In the following two sections we describe the Rank Histogram and the $M V L$ Diagram. In section 5 we compare both for different situations. In the last section we present some conclusions.

\section{Model and prediction system}

We have chosen the so called Lorenz'96 model (Lorenz, 1996; Lorenz and Emanuel, 1998; Wilks, 2005) in order to have spatial chaos and we also introduced parametric noise in order to take into account stochastic fluctuations. It is the same model exploited in Revelli et al. $(2008 \mathrm{a}, 2008 \mathrm{~b})$ to investigate the resonant behavior due to the entanglement between noise and chaos in an extended system. In spite of the fact that it is a kind of toy-model it has a clear relevance to real systems as it is of interest for the analysis of climate behavior and weather prediction (Lorenz and Emanuel, 1998; Orrell, 2003; Wilks, 2005).

\subsection{The Lorenz'96 model}


The model equations are

$$
\dot{x}_{j}(t)=-x_{j-1}\left(x_{j-2}-x_{j+1}\right)-x_{j}+F,
$$

where $x_{j}(t)$ is usually associated with a scalar meteorological quantity such as the geopotential or the temperature, $j=1,2, . . L$ represent the space, and $\dot{x}_{j}$ indicates the time derivative of $x_{j}$. F is the forcing term that we take as fluctuating although it is usually assumed constant.

The Lorenz'96 model has been heuristically formulated as the simplest way to take into account certain properties of global atmospheric models. The terms included in the equation intend to simulate advection, dissipation, and forcing respectively. In contrast with other toy models used in the analysis of extended chaotic systems and based on coupled map lattices, the Lorenz'96 model exhibits extended chaos (when the $F$ parameter exceeds a determinate threshold value $F>9 / 8$ ) with a spatial structure in the form of moving waves. A commonly used parameter is $F=8$, which adjusts the length of these waves to 5 spatial units. If, moreover, $L=40$ the spatial structure mimics geostrophic waves. It is worth noting that the system has scaled variables with unit coefficients, hence the time unit is the dissipative decay time.

Noise was introduced in the model (Revelli et al., 2008a, 2008b) as a spatiotemporally fluctuating parameter $F_{j}(t)=F_{0}+\Psi_{j}(t)$, with $\Psi_{j}(t)$ a spatially white noise dichotomic process. That is, $\Psi_{j}(t)$ adopts the values $\pm \Delta$ with a transition rate $\gamma$ : each state changes according to the waiting time distribution $\varphi_{i}(t) \sim e^{-\gamma t}$. The noise intensity for this process is defined through $\xi=\frac{\Delta^{2}}{2 \gamma}$. The choice of a dichotomic noise is made in order to avoid unnecessary complications (Gutiérrez et al., 1993) as we are dealing with two kinds of noise: a pseudo-random one, generated by chaos, and an external (true) random one introduced in a parametric way. Since the pseudo-random chaotic stationary noise would be quasi-gaussian, the choice of an external noise of a different kind helps us to distinguish between both effects. However, if necessary, one can take the limit of Gaussian white noise, $\Delta \rightarrow \infty, \gamma \rightarrow \infty$ with $\xi$ constant. In order to simulate a meteorological quantity extended around a latitude circle, we consider periodic boundary conditions $x_{0}=x_{L}, x_{-1}=x_{L-1}$. We take $L=128$, instead of the typical value of $L=40$, with the aim of enhancing the strength of chaos.

In this work, as will be explained later, we will assume that the reality is given by the model without noise $F=F_{0}=8$ ( or $\Delta=0$ ), and the modeling incorporates noise. In this 
way we take into account that reality is chaotic and unique and, moreover, models with more or less accuracy can be simulated by varying the intensity of noise.

\subsection{The Ensemble Prediction System}

The Ensemble Prediction System (EPS) seems to be the most appropriate methodology to treat uncertainties in the atmospheric models used in weather forecasting (Molteni, et al., 1996; Palmer et al., 2002). With this method it is possible to use well known deterministic models in a probabilistic framework. Uncertainties due to modeling are accounted for by using ensembles of distinct models (multi-model approach) (Hagedorn et al., 2005) or adding stochastic terms to the weather models. Those due to the propagation and amplification of initial errors are included as members generated with the same model but with distinct perturbed initial conditions.

A typical prediction system based on ensembles contains a control trajectory and ensemble members, which are to be compared with the reality. This is in fact a kind of assimilation of the real data given in a format compatible with the model. On one hand, the control and ensemble trajectories belong basically to the same set of data, both generated by the noisy model. In this work we assume the reality is described as a realization of the Lorenz'96 model without noise $x_{j}(t)$. At a given time $t_{0}$ we prepare the ensemble the initial condition of the control trajectory as identical to reality $x_{c, j}(\tau=0)=x_{j}\left(t_{0}\right)$ and those of each member of the ensemble as a perturbation of the control trajectory $x_{n, j}(\tau=0)=x_{c, j}(\tau=0) \pm \delta x_{n, j}$,

$n=1 \ldots N / 2$. Note that we always include two symmetric initial perturbations $\pm \delta x_{n, j}$ in order to have a symmetric ensemble with $N$ members. The evolution of the ensemble (control included) is evolved for several forecast horizons, $\tau=1,2 .$. , using the Lorenz'96 model with exactly the same realization of noise for each member of the ensemble and the control itself. A set of members $\left\{x_{n, j}(\tau)\right\}$ including a control trajectory $x_{c, j}(\tau)$ is then obtained as forecasts of reality $x_{j}\left(t_{0}+\tau\right)$. In this paper we have used ensembles of $N=100$ members averaging over 100 distinct realizations.

The spread of the ensemble is analyzed by taking as reference either the control trajectory $\delta x_{n, j}(\tau)=x_{n, j}(\tau)-x_{c, j}(\tau)$ or the reality, $\Delta x_{n, j}(\tau)=x_{n, j}(\tau)-x_{j}\left(t_{0}+\tau\right)$. In order to distinguish among them, we will refer to $\delta x$ as perturbations and $\Delta x$ as errors. If the system's evolution occurs without noise, we refer to perturbations, and it corresponds 
to a perfect model case. It is worth stressing that, although we want to give a realistic mimic it is also important to present clearly the separation of effects. On the one hand $\delta x$ is only amplified by chaotic effects. On the other hand $\Delta x$ is only affected by model uncertainties that are amplified by chaos. There would be an intermediate case (adding fluctuations to the initial condition in $\Delta x(0))$ that is not treated in this paper since it does not exhibit new behavior and tends to complicate the situation.

\section{Rank Histograms}

The Rank Histogram (RH) (Harrison et al., 1995; Anderson, 1996; Hamill et al., 1996; Talagrand et al., 1997) (also known as Talagrand Diagram) is an excellent way to detect systematic flaws of an ensemble prediction system. It allows one to detect where a verifying analysis usually falls with respect to the ensemble forecast data (arranged in increasing order at each grid point). Such a diagram is basically built up by depicting, in our case, the state distribution of a given Lorenz variable. To obtain the RH in our EPS scheme we examine the ensemble of forecast values $\left\{x_{n, i}(\tau)\right\}_{t_{s}}$ at a determined spatial point $i$, at time $t_{s}$ and a forecast horizon $\tau x_{i}\left(t_{s}+\tau\right)$. Once the corresponding rank has been determined, the process is repeated, changing the sampling time $t_{s}$, as many times as needed in order to obtain a stable histogram of ranks. In this work we make a standard (analyzing errors in EPS) and a non-standard use of RHs. The non-standard use implies the study of perturbations, that is by adopting as reference the control trajectory $\left\{x_{c, i}(\tau)\right\}_{t_{s}}$ instead of the "real" one $x_{i}\left(t_{s}+\tau\right)$.

\subsection{Definition}

Dealing with a generic case let us indicate with $\bar{U}=\left(u_{1}<u_{2}<\ldots, u_{n}\right)$ the sorted values of the ensemble, and with $\mathcal{V}$ the "reality" (or true state). In practice, as we have an imperfect knowledge of the "reality", we describe it through a probability distribution. This distribution is "calibrated" or "reliable" if the probabilities indicate the "real" likelihood of event occurrence. In case of a finite-sized ensemble, this occurs if the "reality" and the ensemble can be considered samples from the same probability distribution. In such a case

$$
E\left[P\left(\mathcal{V}<u_{i}\right)\right]=\frac{i}{n+1}
$$


where $E\left[P\left(\mathcal{V}<u_{i}\right)\right]$ denotes the expected value of the probability $P$. If we define the bounding ensemble members $u_{o}$ and $u_{n+1}$ such that $E\left[P\left(\mathcal{V}<u_{o}\right)\right]=0$ and $E[P(V<$ $\left.\left.u_{n+1}\right)\right]=1$, then

$$
E\left[P\left(u_{i-1} \leq \mathcal{V}<u_{i}\right)\right]=\frac{1}{n+1}
$$

It is worth noting that the expected value is the same for each one of the $n+1$ ranks relative to the sorted ensemble.

A Rank Histogram is obtained by repeatedly tallying the rank of "reality" respect to the ensemble distribution. Let $\mathcal{R}=\left(r_{1}, r_{2}, \ldots, r_{n+1}\right)$ indicate the $\mathrm{RH}$, with $n+1$ possible intervals. The count at a given interval is obtained from

$$
\sum_{j=1}^{i} r_{j}=\left\langle P\left(\mathcal{V}<u_{i}\right)\right\rangle,
$$

where $\left\langle P\left(\mathcal{V}<u_{i}\right)\right\rangle$ indicates an average over a large sample of statistically independent points. The last equation is equivalent to

$$
r_{j}=\left\langle P\left(u_{i-1} \leq \mathcal{V}<u_{i}\right)\right\rangle
$$

Hence, the population of the $j$ th histogram interval is the fraction of times that the "reality" falls between the sorted ensemble members $j-1$ and $j$.

\subsection{Interpretation of the Rank Histogram}

Rank Histograms are commonly used to assess the reliability of an ensemble forecast in relation to an observation (verification). The advantage of using $\mathrm{RH}$ to compare two given random variables relies on the additional information obtained by the shape of the

histogram. As we have mentioned above, a flat histogram is taken as a sign of reliability, a $\bigcap$-shaped histogram indicates too much variability in the ensemble, and $U$-shaped one denotes a lack of variability (Hamill, 2001). This interpretation is correct when both the forecast and reality are nearly independent random variables, but if we use the RH in a more general case some additional considerations must be added. Simple examples showing the different interpretation of shapes in $\mathrm{RH}$ are presented in (Hamill, 2001). It can be seen how the dependence of variables can change the standard interpretation of $\mathrm{RH}$. For instance, flat or U-shaped histograms can be also due to an effect of conditional bias. In our 
case the analysis of perturbations at the first stages of evolution presents a clear example of dependence between variables of the ensemble $u_{j}=x_{i}^{c}+\delta x_{i}^{j}(0)$ and what is taken as verification, the control $v=u_{j}^{c}$. In this case $E\left[P\left(u_{j}<v\right)\right]=E\left[P\left(\delta x_{i}^{j}(0)<0\right)\right]$, and if the initial perturbation is taken as a Gaussian random variable, the resulting $\mathrm{RH}$ is peaked in the middle of the histogram independently of the degree of dispersion of the ensemble.

\section{Mean-variance of logarithms Diagram}

At this point we consider the Mean Variance of Logarithms or MVL Diagram (Gutiérrez et al., 2008), where the evolution of two indices (indicated below) are displayed along the axis of a two dimensional diagram. Such a diagram is a powerful tool to characterize and graphically represent the evolution of finite perturbations in systems with spatiotemporal chaos. Hence it should be useful to analyze the dynamics of perturbations or errors in an EPS. Perturbations are the differences between each member of the ensemble and the control. When the spatial system is chaotic, as in the case of the atmosphere, small initial perturbations show two distinctive characteristics, exponential growth of the amplitude and spatial localization. Both phenomena can be explained as a manifestation of the chaotic effect of stretching and folding in a functional space, where each spatial point corresponds to a dimension. Spatial localization appears because there are directions (spatial points) more unstable than others. The difference with a case of low dimensional chaos, such as the standard 3-D Lorenz69 model (Lorenz, 1996), is that in spatiotemporal chaos these direction are not fixed, and the localization phenomenon becomes dynamic.

Traditionally, only the amplitude growth has been used to characterize a chaotic system through the Lyapunov exponent. In López et al. (2004) it has been shown how a more complete analysis of finite perturbations requires a knowledge of the spatial localization. The MVL Diagram quantifies both aspects of amplitude growth and spatial localization in a simple scheme.

\subsection{Definition}

When analyzing perturbations of a system with spatiotemporal chaos, it is observed that very quickly they become localized due to the multiplicative character of the growth. This results in log-normal (or generally log-based) statistics. Note that logarithmic normal 
statistics of error growth is a general property of chaotic systems and not a spatial effect, as shown some years ago by Benzi and Carnevale (1998) in low dimensional chaotic systems. Due to this log-normal characteristic (López et al., 2004), the behavior of the logarithm of absolute values of perturbations

$$
h_{i}(t)=\ln \left(\left|\delta x_{i}(t)\right|\right)
$$

was analyzed. The evolution of this quantity results to be similar to the one of kinetic roughening of interfaces defined in space $i$ and time $t$ (Pikovsky and Politi, 1998). This type of processes can be characterized by using the first two moments, mean and variance (spatial mean is represented by an over-line $\ldots$ whereas, as before, $\langle\ldots\rangle$ means averaging over samples).

- Spatial mean of the "interface", given by

$$
\begin{aligned}
M(t) & =\left\langle\overline{h_{i}(t)}\right\rangle=\left\langle\frac{1}{L} \Sigma_{i} h_{i}(t)\right\rangle \\
& =\left\langle\ln \left(\Pi_{i}\left|\delta x_{i}(t)\right|\right)^{1 / L}\right\rangle=\ln \rho(t)
\end{aligned}
$$

evolves in a characteristic linear regime as $M(t) \sim \ln \rho(0)+\lambda t$ (where $\lambda$ is the leading Lyapunov exponent, see (López et al., 2004) for details), until saturation by nonlinear effects.

- Squared width of the interface (roughness) is defined as the variance of perturbations $V(t)$ around the spatial mean

$$
V(t)=\left\langle\overline{\left(h_{i}(t)-\overline{h_{i}(t)}\right)^{2}}\right\rangle
$$

This magnitude grows, as explained below, as a power-law of the form $V(t) \sim t^{2 \beta}$ in the linear regime. In the nonlinear regime the variance decays due to nonlinear effects.

The $M V L$ Diagram is obtained by plotting $V$ against $M$. The mean $M$ is associated with the exponential growth in time. The variance $V$ accounts for the spatial localization. These two magnitudes give useful information about different aspects of the dynamics of growth that can be used to characterize ensembles in a prediction system based on them. Here it is important to remark that, by definition and contrary to what happens in $\mathrm{RH}$, the MVL representation only considers spatial effects of perturbations, without any reference 
to the sampling of the ensemble (note that the index of each member is irrelevant). In this aspect RH and MVL Diagrams are complementary, and as we will see, useful to characterize ensembles.

\subsection{Analogy with the growth of rough interfaces}

The analogy with the growth of rough interfaces provides us with a very sound theory that allows the use of known scaling dynamics laws with universal definitions and the possibility of defining characteristic lengths (Barabasi and Stanley, 1995). A compact picture of the growth of a rough interface, $h(x, t)$, can be obtained by considering the evolution of two characteristic lengths, one horizontal defined as a correlation length $l_{c}(t)$, the other vertical defined as the squared width of the interface $V(t)$.

The spatial correlation length $l_{c}(t)$ can be obtained from a correlation function such as $C(y, t)=\langle\overline{h(x+y, t) h(x, t)}\rangle \sim V(t)-y^{2 \alpha}$, for $y<l_{c}(t)$. The scaling laws of the interfacial growth postulate that the correlation length grows as a power law $l_{c}(t) \sim t^{z}$, zeing the so called dynamic exponent. While the interface grows, the correlated parts of length $l_{c}(t)$ are fractal curves with roughness exponent $\alpha$, so $V(t) \sim l_{c}(t)^{2 \alpha} \sim t^{2 \beta}$, and $\beta=\alpha z$. Finally, the growth process saturates when the correlation length reaches the system size, $l_{c}\left(t_{\text {sat }}\right)=L$ in a saturation time $t_{\text {sat }}$.

These laws indicate that all length scales (horizontal and vertical) and times are related to each other. This is the reason why we can use a vertical length, $V$ the squared width of the interface, as a measure of correlation in the logarithmic perturbation. Moreover, the exponents $\alpha, \beta, z$ are universal. Our interfaces evolve in the so called KPZ universality with values $\alpha=1 / 2, \beta=1 / 3$, and $z=3 / 2$ (Barabasi and Stanley, 1995).

\subsection{Localization of perturbations}

In Fig. 1 we show a typical $M V L$ Diagram corresponding to the evolution of perturbations $\delta x_{i}$ for the Lorenz'96 model. Here, the initial conditions are random with a Gaussian distribution, with a small variance $\sigma=5 \times 10^{-10}$. Hence, we are considering the case of a perfect model with the aim of focusing only on the effects of chaos. Now the noise intensity is zero, reality and control coincide, and also perturbations and errors are identical, by 
definition. The evolution goes from left to right, and each point in the diagram corresponds to a given forecast horizon. $M(\tau)$ gives the logarithmic amplitude, and is a measure of the amplitude growth on logarithmic scale. The variance of the logarithmic fluctuation $V$, corresponds to the squared width of the above defined interface, and its evolution follows the typical scaling laws, growing with $V(\tau) \sim \tau^{2 / 3}$ as an infinitesimal fluctuation in the first part of the curve. The second part, with a decreasing variance (negative slope), corresponds to the effect of the nonlinearities that progressively destroy the acquired correlation.

In order to offer a better picture of the growth of the interface, we show the effect of localization on perturbations and errors, as it is the main distinctive effect emerging from chaos. As this phenomenon is far from being intuitive, on the left hand side of the figure we show snapshots of the perturbation space evolution $\delta x_{i}$ for a generic member of the ensemble at four different representative forecasting times.

Each point (progressing from $(a)$ to $(d)$ ) establishes a typical characteristic evolution time in the MVL Diagram. Points $(a)$ and $(b)$ indicate some representative times for the system evolution on the positive slope part of the curve. That is, in the part of the curve where infinitesimal perturbations effects are relevant, with the linearized equations governing the evolution of the system. Point $(c)$ indicates the maximum of the curve, corresponding with the time associated to the maximum localization of infinitesimal perturbations' growth. Point $(d)$, located on the curve's negative slope part, shows a representative time when nonlinear (finite perturbations) effects are relevant. This is an important phenomenon that appears when considering finite perturbations: the progressive loss of correlation due to nonlinear effects that saturates the growth. Finally, at point $(e)$ the system reaches the asymptotic region and the form of perturbations becomes again Gaussian (not plotted in the figure) similar to the initial point $a$. Note that the $M V L$ Diagram shows clearly the two regimes of infinitesimal and finite fluctuations (Fig.1).

From this picture we can see how an increase of the logarithmic variance $V$ corresponds to a stronger spatial localization in perturbations rather than to an increase in dispersion. This happens in all log-normal (or other log-based) statistical distributions that come from a random multiplicative process.

\subsection{Interpretation of the MVL Diagram}


When using $M V L$ Diagrams to analyze perturbations or errors in EPS three important features can be highlighted.

- The amplitude growth is obtained as $\exp [M(\tau)]$, with $\tau$ the forecast horizon in section 2.2. Into fact, for a large enough $\tau$ we could obtain the main Lyapunov exponent, $M(\tau) \sim \lambda \tau$, but it is not usually the case since saturation by nonlinear effects appears very quickly (López et al., 2004).

- $V(\tau)$ is an index of spatial localization that can be used as a quantifier of the strength of chaos in the presence of noise. Note that $V(\tau)$ is also a robust indicator of the type of statistical distribution of perturbations. For instance, Gaussian perturbations exhibit a characteristic value of $V \sim 1.23$ regardless of the amplitude of the initial perturbation (Gutiérrez et al., 2008).

- The slope of the curve is an indicator of dynamical assimilation. A positive slope indicates increasing localization that occurs when perturbations tend to the attractor. A negative slope means that perturbations are not dynamically compatible with the current flow (Gutiérrez et al., 2008).

In conclusion, MVL Diagrams can be easily calculated in any prediction system based on ensembles, and they can be simply interpreted. They offer information about the initialization procedures and the model formulation which in general constitute a kind of fingerprint of the model. An example of how this technique is used for model inter-comparison in EPS can be found in Fernández et al. (2009).

\subsection{Effects of noise: the MVL of errors}

The $M V L$ Diagram has only been used to analyze perturbations in spatiotemporal chaotic systems. The extension to the analysis of errors is straightforward, simply by taking the interface $h_{i}=\log \left(\Delta x_{i}\right)$ and continuing with the same interpretation. The nontrivial and relevant point is how the effect of noise changes the known pure chaotic picture.

When considering errors with distinct intensities (noise is added to the ensemble evolution) the $M V L$ Diagram changes in such a way that the bell-shape form is retained but with a lower value of the maximum of $V$ than in the $M V L$ Diagram for the case without 
noise. Hence, we can infer that the bell-shape is due to the chaotic characteristic of the model and the reduction of the bell maximum's height is related to the noise effect. This is clearly shown in Figs. 2 and 3 where the $M V L$ diagrams correspond to cases where noise was added to the evolution of the Lorenz'96 model. In order to analyze with more detail the effect of the noise in the right hand panels of Fig.3 we also depict the time evolution of both components, $M(t)$ and $V(t)$. Note that the time origin (not plotted) is the same in all cases and we start plotting the first value of the evolution. Then the first effect of noise appears at this initial time, where the amplitude changes instantaneously. The second effect can be observed in the slope of the amplitude, $\sim M(t) / t$, that is close to the main Lyapunov exponent. This slope becomes smaller for stronger noise. If we associate the strength of chaos to the value of the main Lyapunov exponent, we can say that an effect of noise is to reduce this intensity, a phenomenon known as chaos reduction (Balanov et al, 2003; Choe et al, 2005; Liao et al, 2008). Finally, the saturation time of the amplitude, or the time where the spatial localization (measured by $V$ ) becomes maximum, is also reduced by noise. Note that this time can be related to the forecast time, and then it is an important element in the predictability analysis. As a consequence we can say that the forecast time becomes shorter due to the effect of noise despite having a smaller Lyapunov exponent. This is perceived as a contradiction that occurs due to the initial growth of the amplitude that induces a shorter saturation time.

It is worth remarking here that we are studying a particular case, where the Lorenz'96 parameter $(F)$ for the control and the ensemble are the same as in reality. However, more general situations could be considered, with different parameters, a problem that will be the subject of further work.

\section{Error analysis with RH and $M V L$ Diagrams}

MVL Diagrams provide a good picture of the dynamical evolution of perturbations and errors but they are not sensitive to statistical aspects of the ensemble in relation to sampling. For instance, information about the reliability of ensembles is not attainable from $M V L$ Diagrams. Conversely, RH offer information about the ensemble's reliability while being much less sensitive to the effects of noise. When the diagrams are observed together, one expects to get a very complete characterization of the ensemble evolution, useful for dealing 
with EPS in complex systems.

We illustrate this fact in two interesting situations that analyze, respectively, the effect of noise in a case with random initial conditions, and the use of dynamically assimilated initial conditions in the case of a perfect model. In this way we try to isolate distinct aspects that are important in the treatment of errors. In order to have the joint information from RH and $M V L$ Diagrams we have plotted simultaneously the extreme left $\left(T_{l}\right)$, central $\left(T_{m}\right)$, and extreme right $\left(T_{r}\right)$ values of the $\mathrm{RH}$, with the normalized variance $V^{*}=V / V_{\max }$, versus time, for the above mentioned cases.

\subsection{Deterministic and stochastic noise}

In Fig. 4 we show three cases corresponding to the inclusion of different intensities of noise, and with an uncorrelated Gaussian initial perturbation. The first case, upper panel, is a perfect model situation, without noise. As the only fluctuating effects are due to chaos, the variation of both diagrams' results well synchronized. The time at which localization takes place corresponds to a delta shaped convex diagram, whereas when localization is lost by nonlinear effects, the $\mathrm{RH}$ takes on a flat shape. The delta peaked $\mathrm{RH}$ remains constant over the whole infinitesimal phase, indicating that although perturbations localize and changes its statistics very fast (the slope of $V(t)$ is steeper), they remain independent of the control trajectory. The end of the deterministic effect, given by the time at which $V$ becomes small and stationary, coincides with the beginning of the stochastic time, when the RH becomes almost flat.

In the second case, middle panel in Fig. 4, we include noise but moderate enough to have $\cap$-shapes at the beginning of evolution. In the $\mathrm{RH}$ we can observe three zones in which the shape is markedly convex $(\tau<250)$, lightly convex $(\tau<2500)$, and almost flat $(\tau>2500)$. The course of the deterministic noise, when $V$ increases by chaos and decreases by nonlinearity, is mainly included in the intermediated zone. The end of the deterministic period is also within this zone and this occurs well before the beginning of the stochastic regime. Roughly speaking we could say that the effect of moderate noise consists of a weak suppression of chaos that develops as a loss of spatial localization together with a gain of reliability.

In the third case in lower panel in Fig. 4, the noise is very strong, producing a marked 
U shape in the $\mathrm{RH}$, indicating a complete domination of the noise, until the stochastic time at which the diagram becomes flat. The effect of chaos is completely hidden in the RH but, surprisingly, it is perfectly identified in the $M V L$ Diagram.

\subsection{Localized initial conditions in a perfect model case}

In the previous subsection we have used random initial conditions, and we were able to see in the $M V L$ Diagram how such initial conditions localize due to the effect of the deterministic chaos. This randomness in the initial conditions is a source of uncertainty that can be minimized by using adequately prepared conditions. A good choice is the use of dynamically localized perturbations, coming from either a combination of Lyapunov vectors or an ensemble of bred vectors. In this paper we use logarithmic bred vectors generated as explained in Primo et al. (2008). A breeding cycle is performed by evolving with Eq. (1) two close trajectories and normalizing in order to keep a given amplitude constant (here we adopt $A=10^{-4}$ ). After a number of cycles of breeding, perturbations become stationary and ready to be used as dynamically assimilated initial conditions.

In Fig. 5 we show the $M V L$ Diagram, without noise, for two different cases: using random initial conditions and using dynamically localized initial conditions with bred vectors. The random initial condition case follows a typical trajectory that can be explained, using the analogy with rough interfaces, as the growth of the height $(M)$ and squared width $(V)$ of an interface in the KPZ universality class (Primo et al., 2006). The case of dynamically localized initial conditions is not standard since the localization is not complete and it increases slowly in a relaxational way. Note that with very small initial amplitudes the random perturbation can achieve better localization than the initially localized case. Figure 6 shows in two panels from top to bottom, the time evolution of RH (also including the time dependence of $V^{*}$ ) corresponding to the situation indicated in the previous MVL Diagram. As before, and in order to have the joint information from $\mathrm{RH}$ and $M V L$ Diagrams, in Fig. 6 (and later in Fig. 8) we have plotted simultaneously the extreme left, the central, and the extreme right values of $\mathrm{RH}$, together with the normalized variance $V^{*}$, versus time.

In Fig. 7 we show once again the MVL Diagram, also without noise, for two other different initial conditions: dynamically localized initial conditions using bred vectors and localized but dynamically non compatible initial conditions (non dynamically assimilated). Now, the 
dynamically localized initial conditions are completely saturated, so the localization remains constant until the appearance of the effect of nonlinearities, when the variance $V$ begins to decrease. The non dynamically assimilated initial conditions are obtained by a simple disordering of the previously obtained dynamically assimilated initial conditions. The evolution in this case shows a rapid decrease of variance $V$ due to the lack of compatibility with the dynamics, followed by a gain in localization when the perturbation becomes assimilated. Figure 8, similarly to Fig. 6, shows in panels from top to bottom, the time evolution of the $\mathrm{RH}$ together with the time dependence of $V$, corresponding to the situation indicated in Fig. 7.

The important message here is that all the indicated situations are clearly identified with the variance of $V$, whereas the $\mathrm{RH}$ is insensitive to changes due to the chaotic behavior.

Two important aspects should be remarked. Firstly, it can be observed from Fig. 6 and 8 that when the initial ensemble is dynamically prepared, the $\mathrm{RH}$ is almost flat from the very beginning. This can be understood since an initial prepared ensemble comes from a stationary evolution state that produces statistical independence between members and

the control. The ensemble reproduces or follows the control simulation's evolution, as it is sampled from the same distribution (Smith, 1999).

Hence, with a dynamically prepared initial ensemble we obtain a better spatial resolution (increase of $V$ ) with more reliability (flatter $\mathrm{RH}$ ). Obviously, both effects are a consequence of the elimination of spurious spatial noise. The second remark is related to the ability of the logarithmic variance to detect badly assimilated initial conditions (last panel of Fig. 8). In this figure the initial condition has been localized as in the previous one but the dynamical compatibility was perturbed by a simple disordering of the obtained initial condition. Note that $\mathrm{RH}$ are insensitive to these effects of dynamical assimilation.

Finally, for the sake of completeness, we show in Fig. 9 the MVL diagram in a case of dynamically prepared initial conditions with noise. The effects of noise are the same as in the case of random perturbations, but now, besides the instantaneous initial change of amplitude, there is also a reduction of the spatial localization $(V)$.

\section{Conclusions}

Forecasting in a complex framework with model uncertainties (noise) and chaos (ampli- 
fication of initial perturbations) requires a complete analysis including techniques coming from the theory of chaotic perturbations and sampling. In this paper we propose the joint use of $\mathrm{RH}$ and $M V L$ Diagrams to determine the properties of a simple prediction system based on ensembles. Data is generated in a Lorenz'96 model with controlled noise and mimicking and ensemble prediction system.

RHs inform about the statistical quality of the ensemble (reliability) whereas MVL Diagrams take into account the effect of chaos and noise in the form of spatial resolution and dynamical assimilation. We deal simultaneously with perturbations (simulations without noise) and errors (simulations including noise). Notice that the standard use of RH is for errors and, conversely, MVL Diagrams deal with perturbations. In both cases the extension to a more general use is straightforward.

Although all the analysis in this paper is based on a "toy model" (Lorenz96 model) we expect that the main conclusions will remain valid for more complicated models. That is because the spatial localization phenomenon is a general property of chaotic systems and $V$ is a robust quantifier of spatial localization in any system. In fact, the MVL diagram has been successfully applied to the analysis of realistic weather models, as shown in Fernández et al. (2009).

From the observations of RH and MVL Diagrams applied to perturbations and errors and with more or less dynamically prepared initial conditions we can obtain some general conclusions. The effect of moderate noise is a kind of chaos suppression mechanism that acts to reduce the spatial resolution (reduced localization) and increase the reliability (flatter rank histograms). Strong noise destroys completely the reliability (well formed U-shaped rank histogram). However, the great sensitivity of $M V L$ Diagrams to detect the presence of chaos in a strong noisy environment is outstanding. We conclude by remarking our main result: rank and $M V L$ Diagrams give different information that is useful to optimize the performance of EPS operating on complex systems including spatial chaos and noise.

Acknowledgements: We acknowledge financial support from MEC, Spain, through Grant No. CGL2007-64387/CLI, and also thank the AECID, Spain, for support through projects A/013666/07 and A/018685/08. JAR thanks the MEC, Spain, for the award of a Juan de la Cierva fellowship. HSW thanks to the European Commission for the award of a Marie Curie Chair during part of the development of this work. 


\section{References}

Anderson J.L., 1996: A method of producing and evaluating probabilistic forecasts from ensemble model integrations.; J. Climate 9, 1518-1530.

Anishchenko V.S., Neimann A.B. and Safanova M.A., 1993: Stochastic resonance in chaotic systems ; J. Stat. Phys. 70, 183-196.

Balanov AG, Janson NB and McClintock PVE, 2003: Coherence resonance of the noiseinduced motion on the way to breakdown of synchronization in chaotic systems, Fluct. and Noise Lett. 3 L113-L120.

Barabási A.L. and Stanley H.E., 1995: Fractal Concepts in Surface Growth, Cambridge U.Press, pgs 368.

Benzi R. and Carnevale G.F., 1989: A possible measure of local predictability; J. Atmos. Sci. 46, 3595-3598.

Caroll T.L. and Pecora L.M., 1993: Stochastic Resonance and Crises; Phys. Rev. Lett. 70, 576-579.

Caroll T.L. and Pecora L.M., 1993: Stochastic resonance as a crisis in a period-doubled circuit; Phys. Rev. E 47, 3941-3949.

Choe CU, Hohne K, Benner H, et al., 2005: Chaos suppression in the parametrically driven Lorenz system, Phys. Rev. E 72, 036206.

Gneiting T. and Raftery A., 2005: Atmospheric Science: Weather Forecasting with Ensemble Methods.; Science 310, 248-249.

Gottwald G.A. and Melbourne I., 2005: Testing for chaos in deterministic systems with noise:; Physica D 212, 100-110.

Gutiérrez J.M., Iglesias A. and Rodríguez M.A., 1993: Logistic map driven by dichotomous noise.; Phys. Rev. E 48, 2507-2513.

Gutiérrez J.M., Primo C., Rodríguez M.A., and Fernández J., 2008: Spatiotemporal Characterization of Ensemble Prediction Systems. The MVL Diagram; Nonlin. Processes Geophys. 15 109-114.

Fernández J., Primo C., Cofino A. S.,Gutiérrez J.M., Rodríguez M. A., 2009: MVL Spatiotemporal Analysis for Model Intercorison in EPS. Application to the Demeter Multimodel Ensemble; Climate Dynamics, DOI:10/1007/s00382-008-0456-9.

Hagedorn R., Doblas-Reyes F.J., 2005: The rationale behind the success of multi-model ensembles in seasonal forecasting- I.Basic concept.; Tellus 57A, 219-233. 
Hamill T.M. and Colluci S.J., 1996: Proc. 13th Conf. on Probability and Statistics in the Atmospheric Sciences, Amer. Meteor. Soc. pags. 51-56.

Hamill T.M., 2001: Interpretation of rank histograms for verifying ensemble forecasts; Monthly Weather Rev. 129, 550-560.

Harrison M.S.J., Richardson D.S., Robertson K. and Woodcock A., 1995: UKMO Tech. Rep. Medium-range ensembles using both the ECMWF T63 and unified models - An initial report. U.K. Meteorological Office.; 153, 25pp.

Kalnay E., 2002: Atmospheric Modeling, Data Assimilation and Predictability, Cambridge University Press.

Liao TL, Yan JJ and Hou YY, 2008: Robust chaos suppression for the family of nonlinear chaotic systems with noise perturbation, Nonlinear Anal.-Theory Meth. \& Applic. 69, 14-23.

López J.M., Primo C., Rodríguez M.A., and Szendro I., 2004: Scaling properties of growing noninfinitesimal perturbations in space-time chaos.; Phys. Rev. E. 70, 056224.

Lorenz E.N., 1996: The essence of chaos, U.Washington Press.

Lorenz E.N. and Emanuel K.A., 1998: Optimal sites for supplementary weather observations: Simulations with a small model.; J. Atmosph. Sci. 55, 399-414.

Molteni F., Palmerand T., Petroliagis T., 1996: The ECMWF Ensemble Prediction System: Methodology and Validation.; Q. J. Roy. Meteorol. Soc. 122, 73-119.

Orrell D., 2003: Model error and predictability over different timescales in the Lorenz 96 systems.; J. Atmosph. Sci. 60, 2219-2228.

Palmer N.T., 2002: The economic value of ensemble forecasts as a tool for risk assessment. From days to decades.; Q. J. Roy. Meteorol. Soc. 128, 747-774.

Pikovsky A. and Politi A., 1998: Dynamic localization of Lyapunov vectors in spacetime chaos; Nonlinearity 11, 1049-1062.

Primo C., Szendro I.G., Rodríguez M.A. and López J.M, 2006: Dynamic scaling of bred vectors in spatially extended chaotic systems.; Europhys. Lett. 76, 767-772.

Primo C., Rodríguez M.A. and Gutierrez J.M., 2008: Logarithmic bred vectors. A new ensemble method with adjustable spread and calibration time, J of Geophys. Res. 113, D04116.

Revelli J.A., Rodriguez M.A. and Wio H.S., 2008: Resonant phenomena in extended chaotic systems subject to external noise: the Lorenz'96 model case.; Physica A 387, 31033110 . 
Revelli J.A., Rodriguez M.A. and Wio H.S., 2008: Noise effects in extended chaotic system: study of the Lorenz'96 model, in Progress in Statistical Mechanics Research, J.S. Moreno Ed. (Nova Science Pub., NY) pgs. 413-425.

Smith L. A., 1999: Disentangling uncertainty and error: On the probability of nonlinear systems, Nonlinear Dynamics and Statistics. Alistair E. Mees. Ed. Birkhauer Press, 31-64.

Talagrand O., Vautard R. and Strauss B., 1997: Proc. ECMWF Workshop on Predictability, ECMWF, Reading, 1-25.

Wilks D.S., 2005: Effects of stochastic parametrization in Lorenz'96 system, Quaterly J. of the Royal Meteor. Soc. B 131, 389-407. 


\section{Figure Captions}

Figure 1: Right: $M V L$ Diagram where five typical times, described in the text. Left: four snapshots of the system's amplitudes associated to the points indicated on the right. Note the different vertical scales in the four cases. Simulations are without noise (perfect model) and the initial condition corresponds to a random Gaussian distribution with $\sigma=5 \times 10^{-10}$.

Figure 2: Right: $M V L$ Diagram where four typical times are indicated. Left: snapshots of the system's amplitudes associated to the points indicated on the right. The parameters are the same as in Fig. (1), but now including noise with $\Delta=10^{-5}$ and $\gamma=10$.

Figure 3: Left: $M V L$ Diagrams for different noise intensities with $\gamma=10$. Open circles $\Delta=0$, black squares $\Delta=10^{-4}$, crosses $\Delta=10^{-1}$. Initial conditions are the same in all three cases, and as in the previous figures.Right: Evolution of $M(t)$ and $V(t)$. Note that the initial point is not plotted.

Figure 4: Left: Rank Histograms at three separate times, and right time behavior for three positions in the histogram with the normalized variance $V^{*}=V / V_{m}$ (rhs). Left: crosses for $t=64$, black squares for $t=960$, and open circles for $t=2048$. Right: continuous line for $T_{l}$ (position 1), dash-dotted line for $T_{m}$ (position 50), and dashed line for $T_{r}$ (position 100). $V^{*}$ is shown with a thick continuous line. Parameters as in Fig. 2. From top to bottom $\Delta=0, \Delta=10^{-4}, \Delta=10^{-1}$.

Figure 5: $M V L$ Diagrams for different initial conditions. Same parameters as in Fig. 1, also without noise. The different curves correspond to: open circles with random initial conditions; black squares to dynamically localized initial conditions using bred vectors.

Figure 6: Evolution of $T_{l}, T_{m}, T_{r}$ and $V^{*}$ with the same lines as in Fig. 4 and without noise. From top to bottom: random initial conditions and the localized initial conditions after breeding.

Figure 7: MVL Diagrams for different initial conditions. Same parameters as in Fig. 1, also without noise. The different curves correspond to: open circles to dynamically localized initial conditions using bred vectors (assimilated), black squares to localized but not dynamically compatible initial conditions.

Figure 8: Evolution of $T_{l}, T_{m}, T_{r}$, and $V^{*}$ with the same lines as in Fig. 4 and without noise. Upper panel: Dynamically assimilated initial conditions after breeding; lower panel: localized but non-assimilated initial conditions. 
Figure 9: $M V L$ Diagrams for different noise intensities with $\gamma=10$. Open circles $\Delta=0$, black squares $\Delta=10^{-4}$, crosses $\Delta=10^{-1}$. Initial conditions are the same in all three cases, now dynamically assimilated after breeding. Note that the initial point is not plotted. 

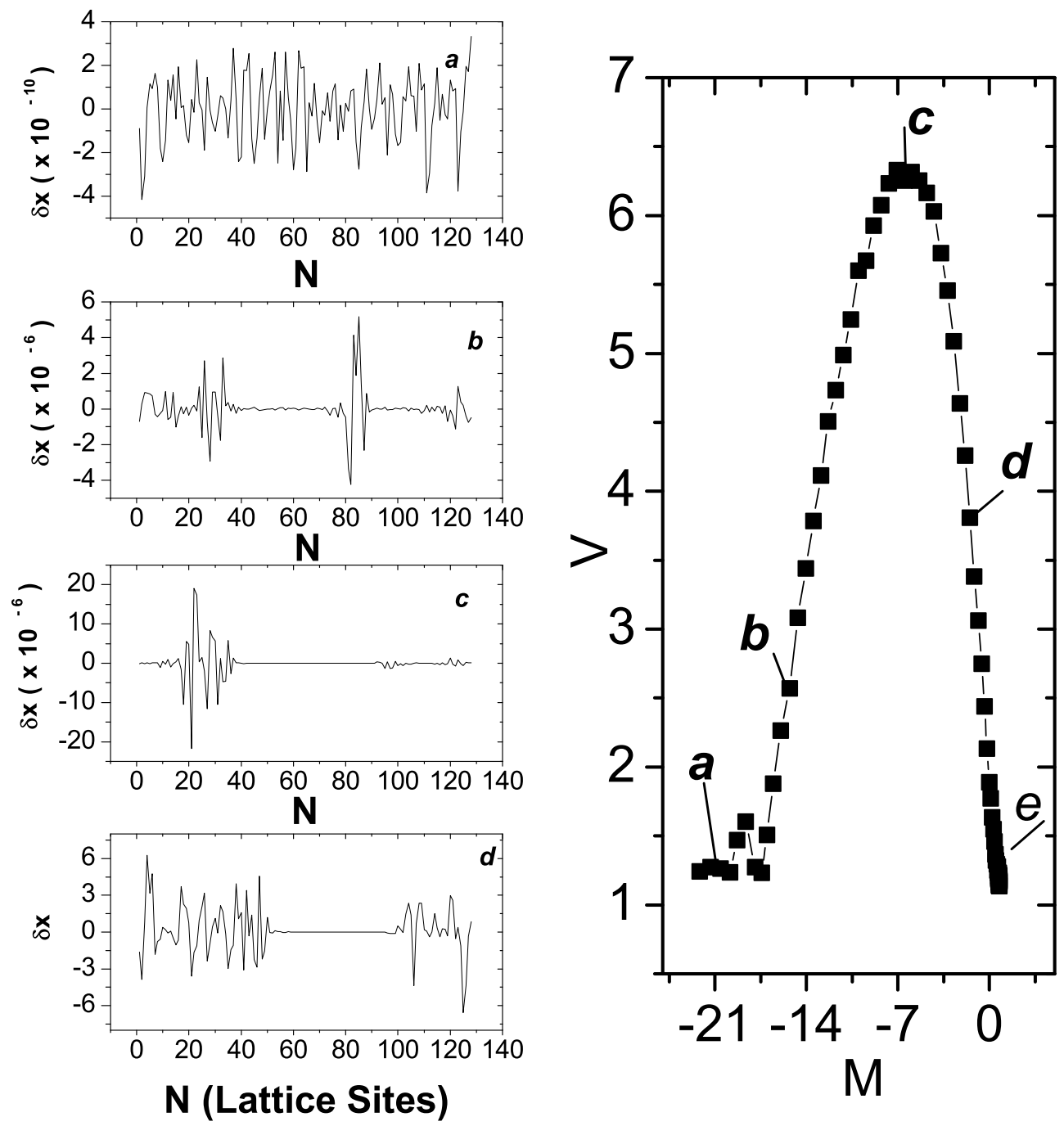

FIG. 1: Right: $M V L$ Diagram where five typical times, described in the text. Left: four snapshots of the system's amplitudes associated to the points indicated on the right. Note the different vertical scales in the four cases. Simulations are without noise (perfect model) and the initial condition corresponds to a random Gaussian distribution with $\sigma=5 \times 10^{-10}$. 

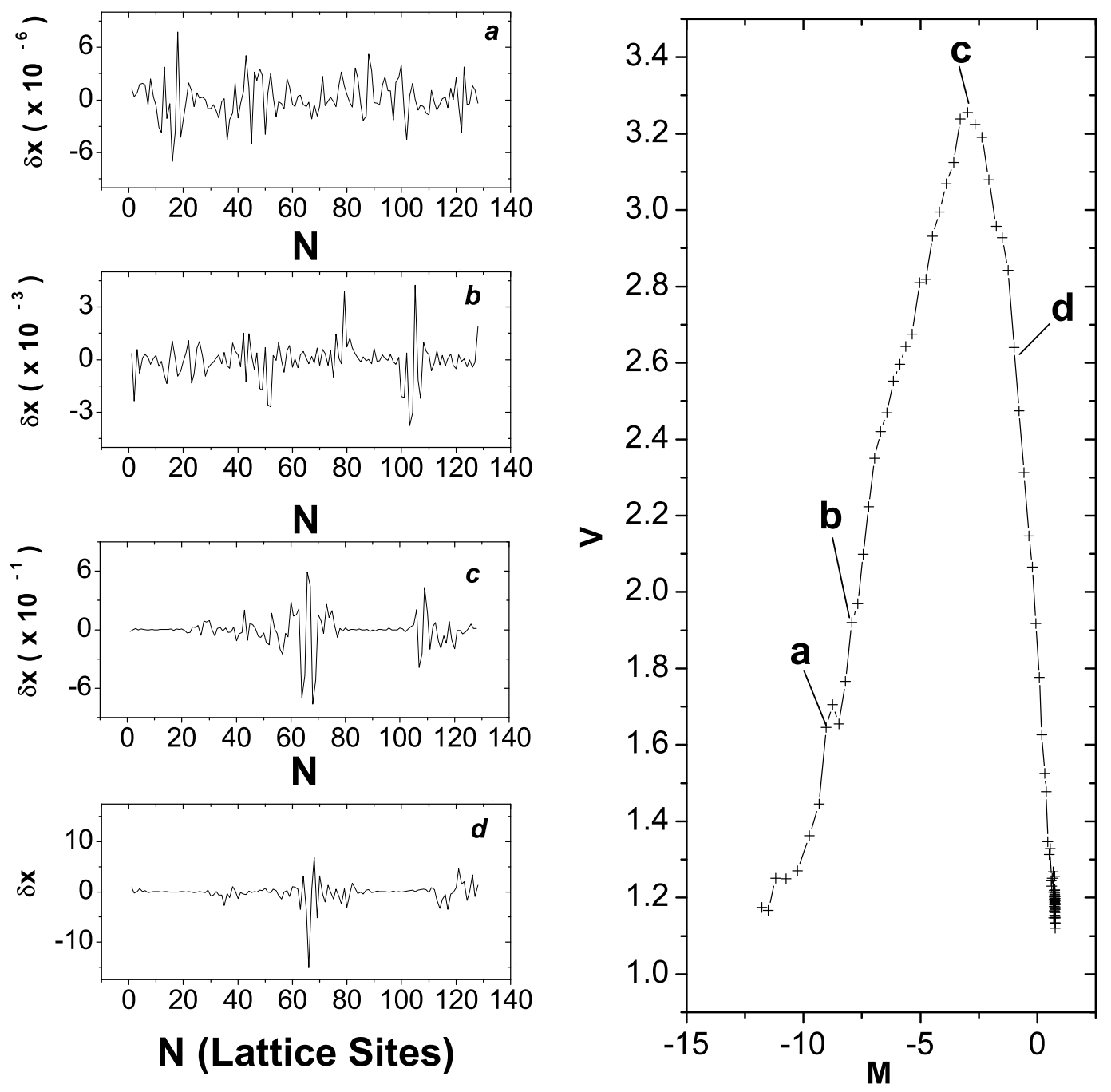

FIG. 2: Right: $M V L$ Diagram where four typical times are indicated. Left: snapshots of the system's amplitudes associated to the points indicated on the right. The parameters are the same as in Fig. (1), but now including noise with $\Delta=10^{-5}$ and $\gamma=10$. 

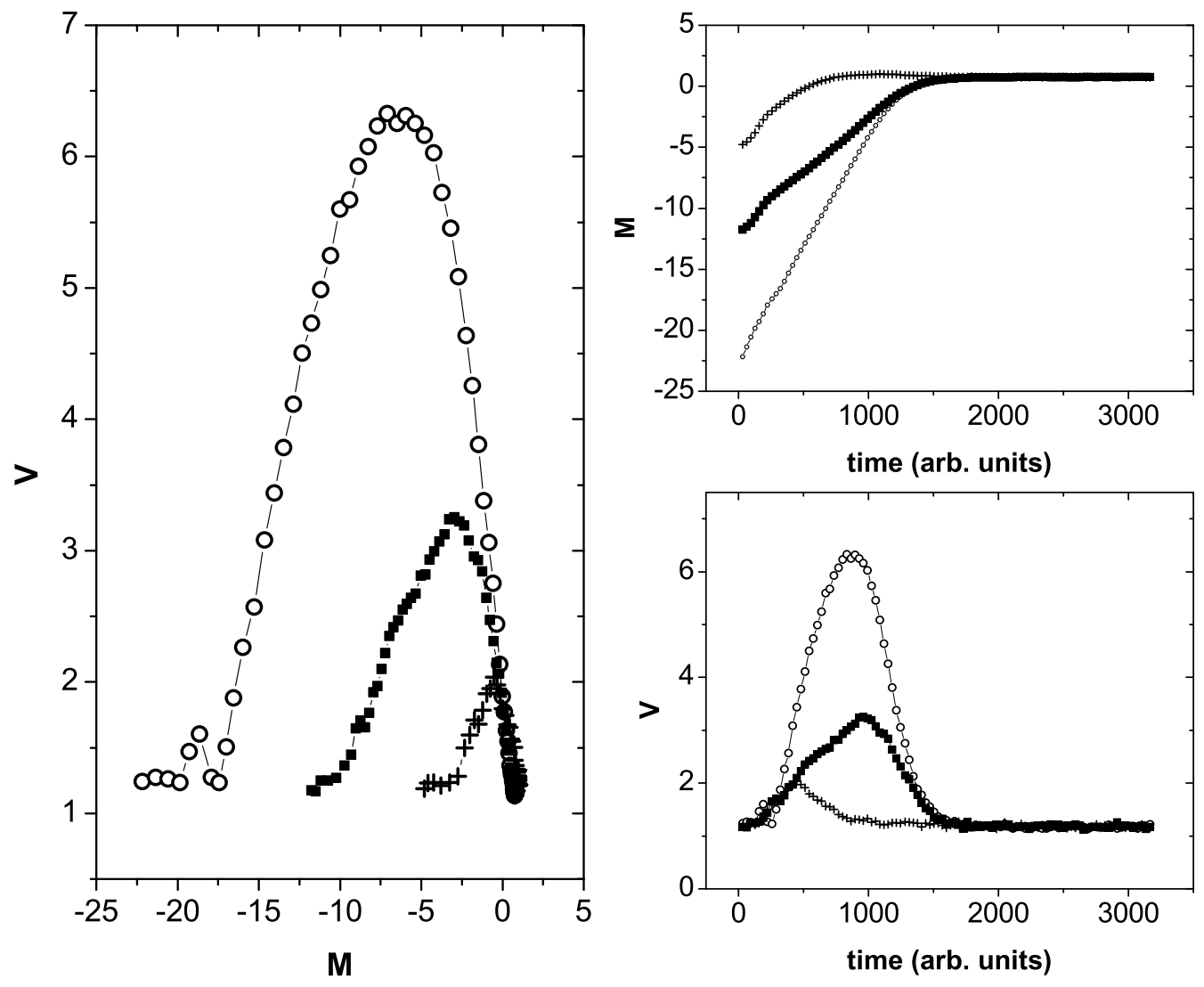

FIG. 3: Left: $M V L$ Diagrams for different noise intensities with $\gamma=10$. Open circles $\Delta=0$, black squares $\Delta=10^{-4}$, crosses $\Delta=10^{-1}$. Initial conditions are the same in all three cases, and as in the previous figures.Right: Evolution of $M(t)$ and $V(t)$. Note that the initial point is not plotted. 

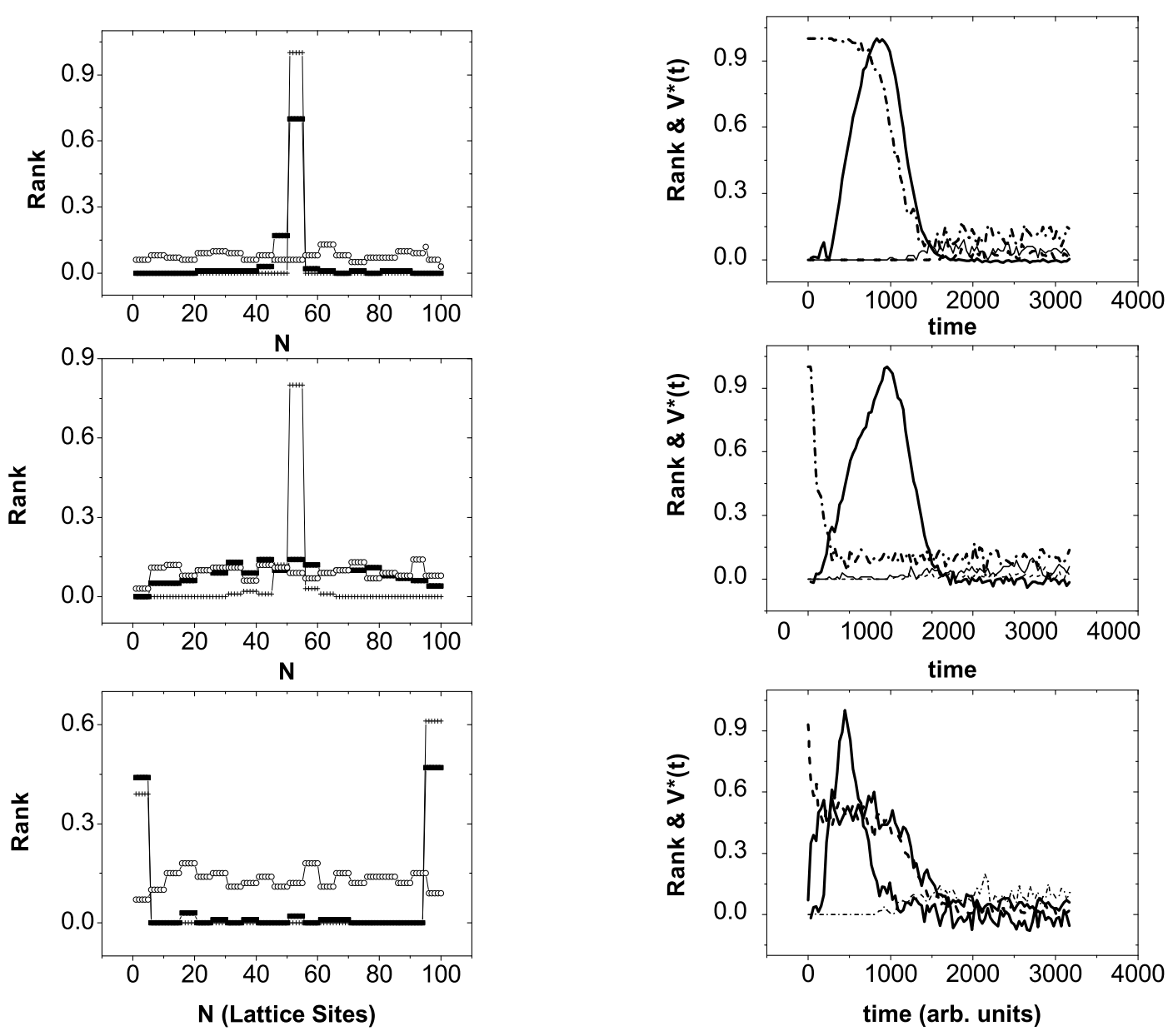

FIG. 4: Left: Rank Histograms at three separate times, and right time behavior for three positions in the histogram with the normalized variance $V^{*}=V / V_{m}$ (rhs). Left: crosses for $t=64$, black squares for $t=960$, and open circles for $t=2048$. Right: continuous line for $T_{l}$ (position 1 ), dash-dotted line for $T_{m}$ (position 50), and dashed line for $T_{r}$ (position 100). $V^{*}$ is shown with a thick continuous line. Parameters as in Fig. 2. From top to bottom $\Delta=0, \Delta=10^{-4}, \Delta=10^{-1}$. 


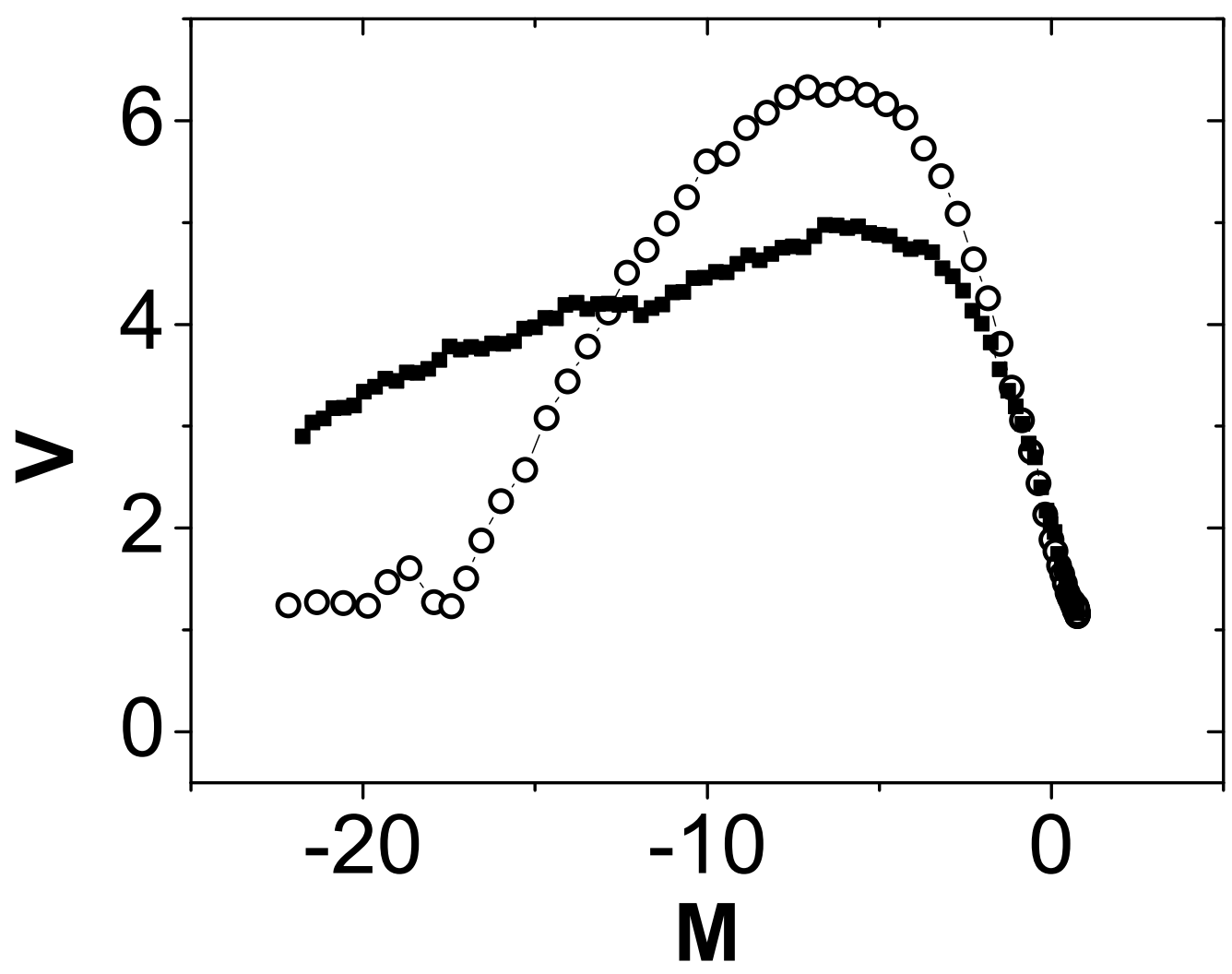

FIG. 5: $M V L$ Diagrams for different initial conditions. Same parameters as in Fig. 1, also without noise. The different curves correspond to: open circles with random initial conditions; black squares to dynamically localized initial conditions using bred vectors. 

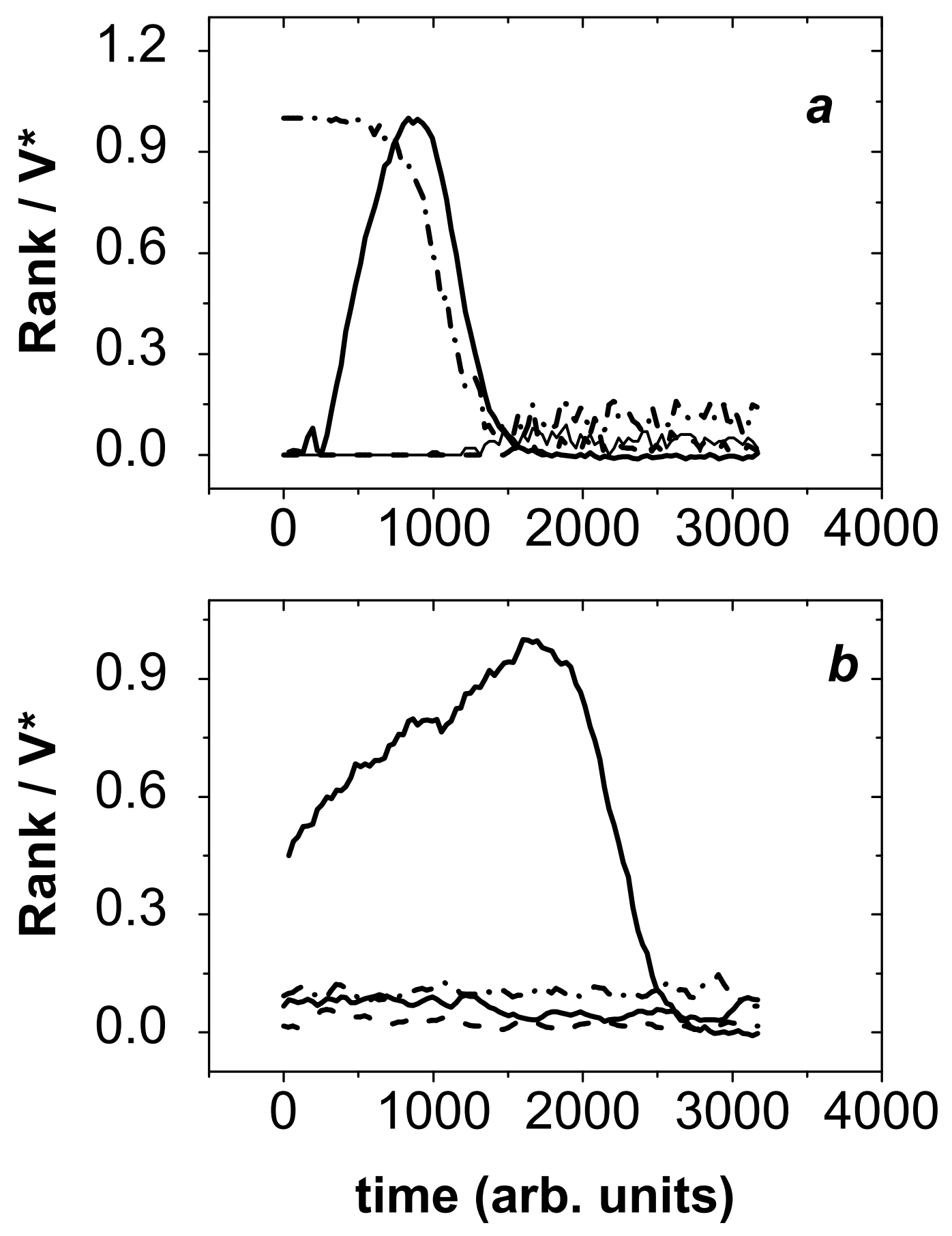

FIG. 6: Evolution of $T_{l}, T_{m}, T_{r}$ and $V^{*}$ with the same lines as in Fig. 4 and without noise. From top to bottom: random initial conditions and the localized initial conditions after breeding. 


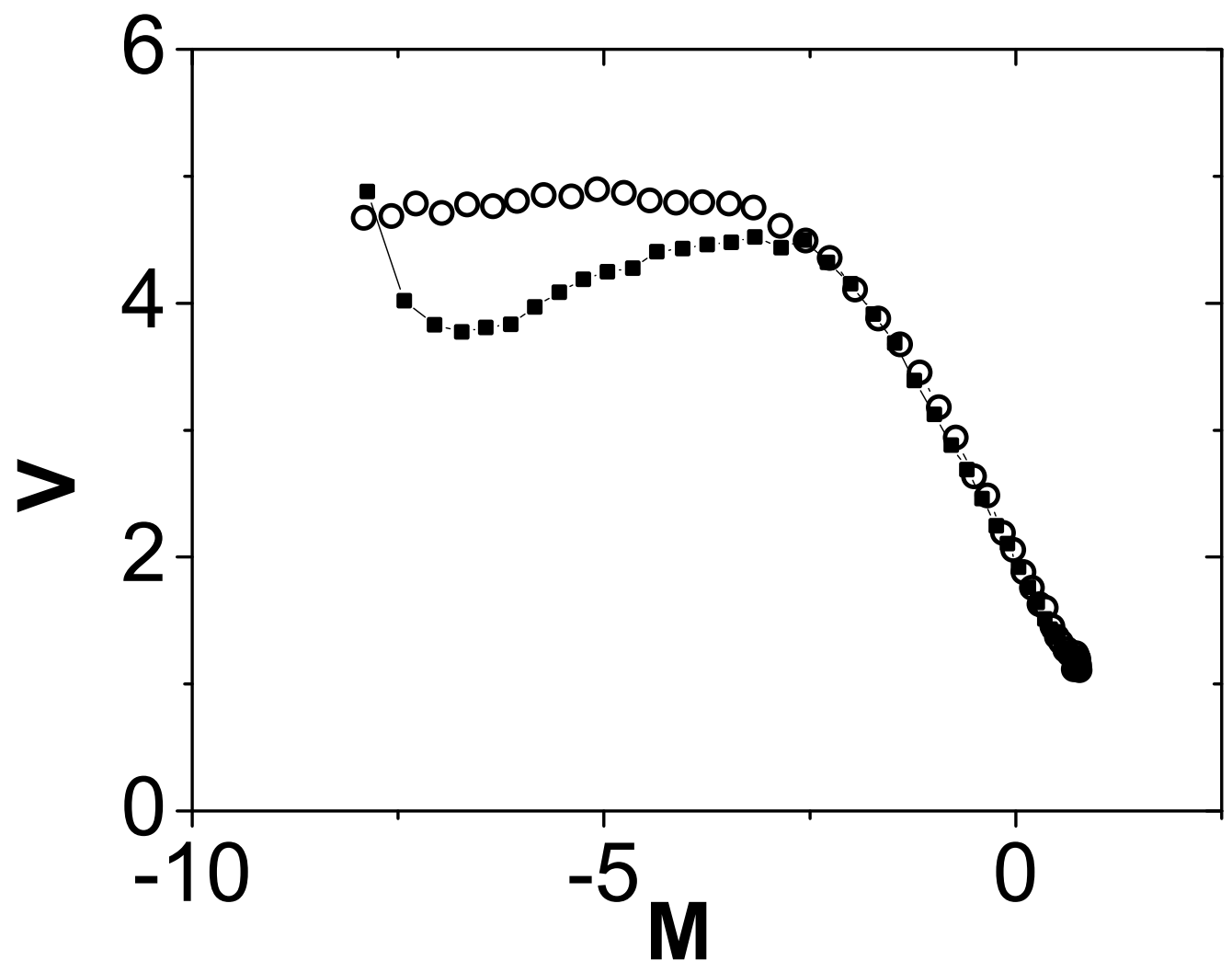

FIG. 7: $M V L$ Diagrams for different initial conditions. Same parameters as in Fig. 1, also without noise. The different curves correspond to: open circles to dynamically localized initial conditions using bred vectors (assimilated), black squares to localized but not dynamically compatible initial conditions. 


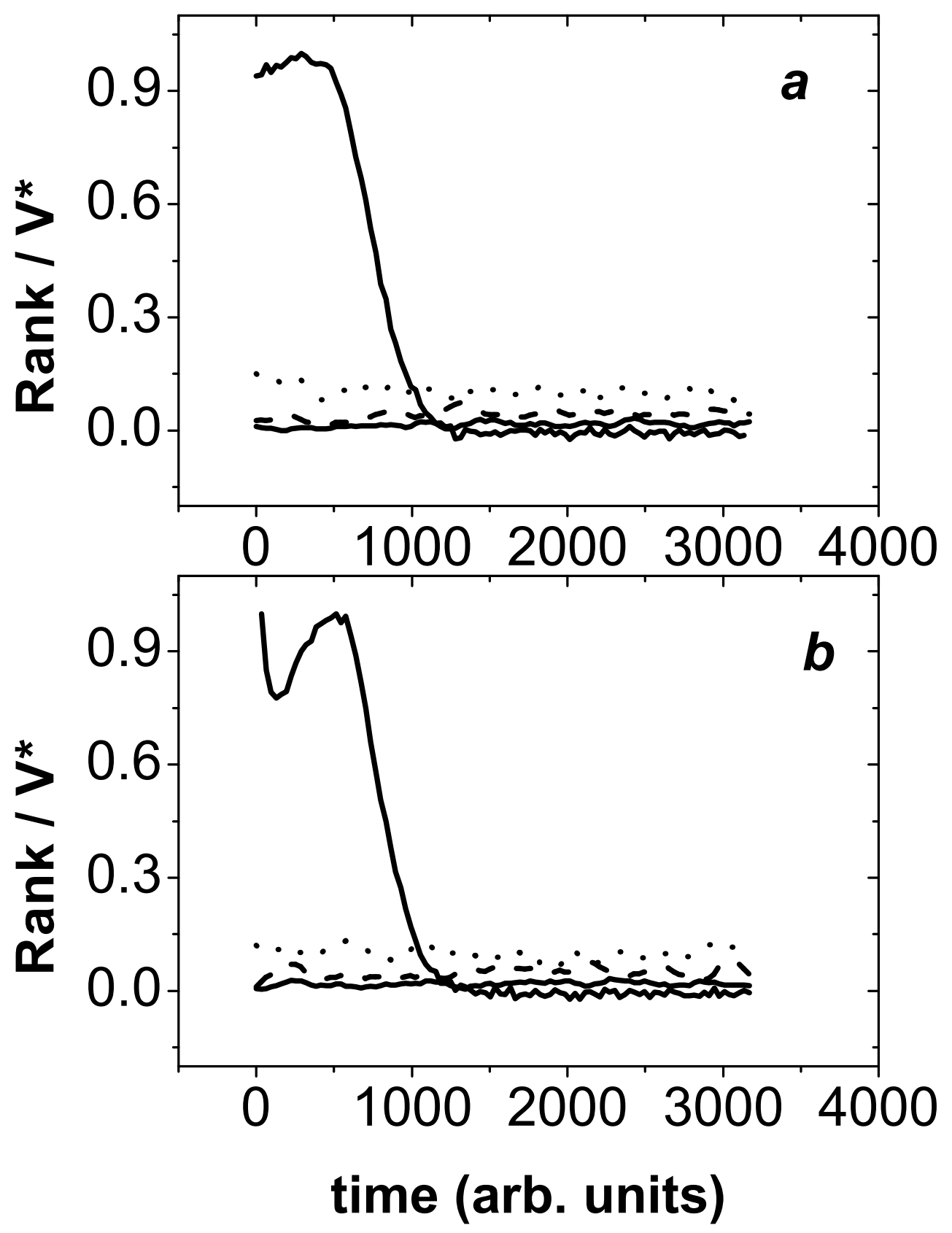

FIG. 8: Evolution of $T_{l}, T_{m}, T_{r}$, and $V^{*}$ with the same lines as in Fig. 4 and without noise. Upper panel: Dynamically assimilated initial conditions after breeding; lower panel: localized but non-assimilated initial conditions. 


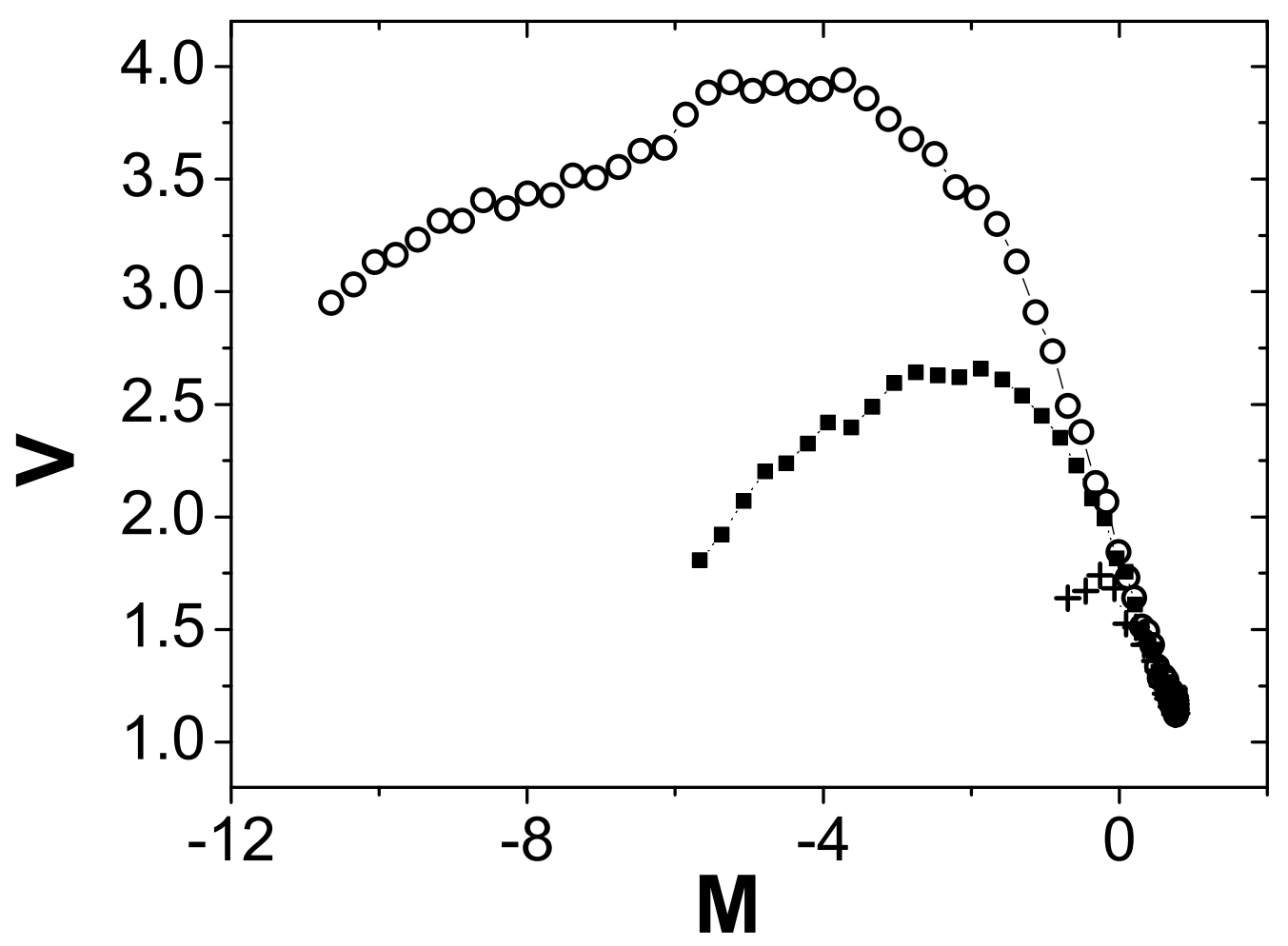

FIG. 9: $M V L$ Diagrams for different noise intensities with $\gamma=10$. Open circles $\Delta=0$, black squares $\Delta=10^{-4}$, crosses $\Delta=10^{-1}$. Initial conditions are the same in all three cases, now dynamically assimilated after breeding. Note that the initial point is not plotted. 